\title{
Genome-wide association analysis of copy number variations in subarachnoid aneurysmal hemorrhage
}

\author{
Joon Seol Bae ${ }^{1}$, Hyun Sub Cheong ${ }^{2}$, Byung Lae Park ${ }^{2}$, Lyoung Hyo Kim², Tae Joon Park ${ }^{1}$, Jason Yongha Kim¹,
} Charisse Flerida A Pasaje ${ }^{1}$, Jin Sol Lee ${ }^{1}$, Tailin Cui ${ }^{3}$, Ituro Inoue ${ }^{3}$ and Hyoung Doo Shin ${ }^{1,2}$

Subarachnoid aneurysmal hemorrhage (SAH) due to cerebral aneurysm rupture is a very serious disease resulting in high mortality rate. It has been known that genetic factors are involved in the risk of SAH. A recent breakthrough in genomic variation called copy number variation (CNV) has been revealed to be involved in risks of human diseases. In this study, we hypothesized that CNVs can predict the risk of SAH. We used the Illumina HumanHap300 BeadChip (317 503 markers) to genotype 497 individuals in a Japanese population. Furthermore, individual CNVs were identified using signal and allelic intensities. The genetic effect of CNV on the risk of SAH was evaluated using multivariate logistic regression controlling for age and gender in 187 common CNV regions (frequency $>1 \%$ ). From a total of 4574 individual CNVs identified in this study ( 9.7 CNVs per individual), we were able to discover 1644 unique CNV regions containing 1232 genes. The identified variations were validated using visual examination of the genoplot image, overlapping analysis with the Database of Genomic Variants (73.2\%), CNVpartition (72.4\%) and quantitative PCR. Interestingly, two CNV regions, chr4:153210505-153212191 (deletion, 4q31.3, $P=0.0005, P^{\text {corr }}$ (corrected $P$-value) $=0.04$ ) and chr10:6265006-6267388 (duplication, 10p15.1, $P=0.0006, P^{\text {corr }}=0.05$ ), were significantly associated with the risk of SAH after multiple testing corrections. Our results suggest that the newly identified CNV regions may contribute to SAH disease susceptibility.

Journal of Human Genetics (2010) 55, 726-730; doi:10.1038/jhg.2010.97; published online 12 August 2010

Keywords: copy number variation; genetic epidemiology; genome-wide CNV association study; genomics; subarachnoid aneurysmal hemorrhage

\section{INTRODUCTION}

Subarachnoid aneurysmal hemorrhage (SAH) due to cerebral aneurysm rupture is a very serious disease that affects $10-15$ out 100000 people. This condition accounts for $6-8 \%$ of the total stroke cases and the mortality rate of affected individuals has been reported to be around $40-50 \% .^{1,2}$ It is known that both environmental and genetic factors are related to $\mathrm{SAH}$, but the exact mechanism that accounts for the development of the disease is still unknown. ${ }^{3,4}$

Copy number variation (CNV) is another form of structural variation. It is defined as a large DNA fragment that ranges from $1 \mathrm{~kb}$ to several megabases and it contains deletions and duplications. ${ }^{5,6}$ $\mathrm{CNVs}$ may contribute to disease susceptibility by influencing the gene expression level. ${ }^{7,8}$ Recent studies have revealed significant associations between CNVs and susceptibility or resistance to human diseases, including autism, inflammatory autoimmune disorders, lung cancer and schizophrenia. ${ }^{9-12}$ Although it has already been acknowledged that CNVs contribute to various complex human diseases, there were no previous attempts to study the relationship between SAH and $\mathrm{CNV}$.
In our previous study, which was focused on the identification of a multiallelic single-nucleotide polymorphism (SNP) marker, we found 597 SNP markers showing a multiallelic CNV genotype, commonly known as the common deletion polymorphism, within the CNV region, and performed a further case-control association analysis. ${ }^{13}$ In this study, however, we used consecutive markers to analyze the identified CNV region covering. To find individual CNVs, we used the pennCNV algorithm, a program that incorporates multiple factors, including signal intensity (log $\mathrm{R}$ ratio (LRR)) and allelic intensity (B-allele frequency), to investigate on more robust risk-associated CNVs. This is the first genome-wide association study to investigate the relationship between common CNV and SAH.

\section{MATERIALS AND METHODS}

Subjects and whole-genome SNP genotyping

All individuals included in this study were of Japanese ethnic origin. The subjects who were recruited from Tokai University donated blood for genetic tests after signing the informed consent that was approved by the institutional review board. The control subjects were screened for not harboring intracranial

${ }^{1}$ Laboratory of Genomic Diversity, Department of Life Science, Sogang University, Shinsu-dong, Mapo-gu, Seoul, Republic of Korea; ${ }^{2}$ Department of Genetic Epidemiology, SNP Genetics Inc., WooLim Lion's Valley, 371-28, Gasan-Dong, Geumcheon-Gu, Seoul, Republic of Korea and ${ }^{3}$ Division of Molecular Life Science, School of Medicine, Tokai University, Shimokasuya 143, Isehara, Kanagawa, Japan

Correspondence: Professor HD Shin, Laboratory of Genomic Diversity, Department of Life Science, Sogang University, Shinsu-dong, Mapo-gu, Seoul 121-742, Republic of Korea. E-mail: hdshin@sogang.ac.kr or Professor I Inoue, Division of Molecular Life Science, School of Medicine, Tokai University, Shimokasuya 143, Isehara, Kanagawa 259-1193, Japan. E-mail: ituro@is.icc.u-tokai.ac.jp

Received 7 June 2010; revised 13 July 2010; accepted 14 July 2010; published online 12 August 2010 
aneurysm using neuroradiological imaging such as digital subtraction angiography, ${ }^{14}$ three-dimensional computed tomography angiography or magnetic resonance angiography. There were no recruitment restrictions with regard to gender. The presence of SAH was confirmed by conventional angiography, three-dimensional computed tomography, ${ }^{15}$ magnetic resonance angiography or surgical finding, whichever is applicable. The study group consisted of 191 patients with SAH and 282 controls without SAH. The control group consisted of individuals who did not have a smoking history and had negative findings on brain computed tomography or magnetic resonance imaging. The age of those in the patient group and control group ranged from 26 to 84 years (mean age $=54.8$ years, s.d. $=11.0$ ) and from 42 to 92 years (mean age $=64.4$ years, s.d.=10.4), respectively. Genome-wide SNP genotyping was performed using the Illumina HumanHap300 BeadChip containing 317503 markers (Illumina, San Diego, CA, USA). Approximately 750 ng of genomic DNA extracted from the blood of all individuals was used to genotype each sample. The assay procedure has been described in our previous study. ${ }^{13}$ The overall SNP genotyping call rate in this study was $99.80 \%$, an indication of high-quality data.

\section{Identification and mapping of individual CNVs}

The signal intensity (LRR) and allelic intensity (B-allele frequency) ratios of all samples were exported from the Illumina BeadStudio software. Samples that did not satisfy the following criteria were excluded from the study: (i) call rate $>99.0 \%{ }^{16}$ (ii) number of identified CNVs $<100$; (iii) s.d. ${ }^{14}$ of LRR $<0.24$, as samples with LRR s.d. $>0.24$ are of low quality, resulting in false-positive CNVs. To identify individual CNVs, we incorporated multiple factors including LRR, B-allele frequency, marker distance and population frequency of the B allele using pennCNV. ${ }^{17,18}$ For verification purposes of the identified CNVs, the CNVpartition program with default criteria (Illumina) was initially used to identify CNVs, after which, results were compared with those obtained using pennCNV. We used karyoview of Ensemble to draw the maps of the identified genomic variations (http://apr2006.archive.ensembl.org/Homo_sapiens/ karyoview). The input data of each variation were prepared following this format: chromosome, start position, end position and status. The input data of all variants were fixed to 'paste file content' menu before the map was drawn. In the case of the $\mathrm{X}$ chromosome, automatic clustering using Illumina BeadStudio software was performed in males and females. To generate a gender file, an automatic estimation of gender was performed using Illumina BeadStudio 3.2 software. Thereafter, the '-chrX' argument for identifying individual CNVs in the $\mathrm{X}$ chromosome was used.

\section{Validation of CNV by visual examination using genoplot image}

Illumina BeadStudio software provides visual genoplot images representing signal intensity ( $y$ axis) and allelic intensity ( $x$ axis) simultaneously per marker. To validate the existence of identified CNV regions, we visually inspected the consecutive changes in signal intensity and allelic intensity at each genoplot image of the marker.

\section{Validation of CNV region by real-time quantitative PCR}

We designed a specific amplification primer set (forward primer sequence: $5^{\prime}$ GTGTCTGAGTGTGGGAACCA-3'; reverse primer sequence: $5^{\prime}$-TCCTGAACT TTGTCCCATCTGAGA- $3^{\prime}$ ) and TaqMan-specific probe (5'-CCAGGCCCATGA TATTCCAC- $3^{\prime}$ ) to validate the existence of the CNV region within chr4:153210505-153212191, the region commonly associated with the risk of $\mathrm{SAH}$. Copy number determination analysis was performed using the ABI Prism 7900 sequence detection system. The RNaseP gene was coamplified with the marker, which was then used as an internal standard. Amplification reactions $(10 \mu \mathrm{l})$ were carried out using $10 \mathrm{ng}$ of template DNA, $1 \times$ TaqMan Universal Master Mix buffer (Applied Biosystems, Foster City, CA, USA), 900 nм of each primer and $250 \mathrm{~nm}$ of each fluorogenic probe. Thermal cycling was initiated with $2 \mathrm{~min}$ incubation at $50^{\circ} \mathrm{C}$, followed by a first denaturation step of $10 \mathrm{~min}$ at $95^{\circ} \mathrm{C}$, and then $40 \mathrm{cycles}$ of $15 \mathrm{~s}$ at $95^{\circ} \mathrm{C}$ and for $1 \mathrm{~min}$ at $60^{\circ} \mathrm{C}$. Three replicate reactions were performed for similar primer pairs and each copy number of individuals was calculated by Copy Caller v1.0 (Applied Biosystems) using the comparative $C_{\mathrm{t}}$ method.

\section{Statistical analysis and gene ontology analysis}

We generated input data of both loss (homozygous deletion=0X; hemizygous deletion $=1 \mathrm{X}$; normal copy number $=2 \mathrm{X}$ ) and gain (normal copy number $=2 \mathrm{X}$; duplication $=3 \mathrm{X}$; amplification $=>4 \mathrm{X}$ ). In the case of loss, $\mathrm{CNV}$ status $0 \mathrm{X}, 1 \mathrm{X}$ and $2 \mathrm{X}$ were coded with 'A_A', 'A_B' and 'B_B', respectively. In the case of gain, CNV status $>4 \mathrm{X}, 3 \mathrm{X}$ and $2 \mathrm{X}$ were coded with 'A_A,' 'A_B' and 'B_B,' respectively. Logistic regression analyses controlling for age (continuous value) and sex $($ male $=0$ or female $=1)$ as covariate were used to calculate the $P$-values for case-control analysis. Golden HelixTree software (Golden Helix, Bozeman, MT, USA; http://www.goldenhelix.com) was used for the association analyses and $\chi^{2}$-tests were used to determine whether the individual variants were in Hardy-Weinberg equilibrium.

To correct for multiple testing, the effective number of independent markers representing common CNVs (frequency $>1 \%$ ) was calculated using the software SNPSpD (http://genepi.qimr.edu.au/general/daleN/SNPSpD/), which is based on the spectral decomposition of matrices of pairwise linear disequilibrium values between markers. ${ }^{19}$ The number of independent marker loci was calculated as 89.3639 , and this was applied to correct for multiple testing $(P$-value $\times 89.3639)$. To provide insights into the functional enrichment of CNVs, we performed gene ontology (GO) analysis using GOstat (http:// gostat.wehi.edu.au) provided by Tim Beißbarth. ${ }^{20,21}$

\section{RESULTS}

In this study, we identified 4574 individual CNVs and $1644 \mathrm{CNV}$ regions in a Japanese population sample $(n=473)$ using the Illumina HumanHap300 BeadChip. The average number of CNVs per sample was 9.7, with an average length and median size of 63.5 and $31.9 \mathrm{~kb}$, respectively (Supplementary Table 1). Furthermore, results revealed that $1644 \mathrm{CNV}$ regions identified in this study contained 1232 genes. Among the common $\mathrm{CNV}$ regions, 187 had a frequency of $>1 \%, 66$ $\mathrm{CNV}$ regions had $>2.5 \%$ and $22 \mathrm{CNV}$ regions had $>5 \%$. Moreover, the 22 common $\mathrm{CNV}$ regions that contained 84 genes along with the rest of the regions were found to overlap with previously reported CNVs in the Database of Genomic Variants (DGVs) (Table 1). A karyotype map that shows the identified CNV regions according to their frequency is shown in Supplementary Figure 1. Common CNV regions with a frequency of over $1 \%$ were distributed evenly on the chromosomes, including X chromosomes. Furthermore, we were able to observe that $73.2 \%$ of the CNVs identified in this study matched with those found in DGV. All of them overlapped with previously reported CNVs in DGV. Supplementary Figure 2 shows the size distribution of individual CNVs identified in this study. Most of the identified CNVs were distributed within $1-50 \mathrm{~kb}$ in range.

To determine the association between the identified CNVs and the risk of $\mathrm{SAH}$, we performed logistic regression analyses controlling for age and sex as covariates in 187 common CNV regions (frequency $>1 \%$ ). Results showed that two CNV regions (chr4:153210505153212191, $P=0.0005$, and chr10:6265006-6267388, $P=0.0006$ ) were significantly associated with the risk of SAH (Table 2). After performing Hardy-Weinberg equilibrium test on the two CNV regions, we found no significant differences between the distribution of the observed genotypes and the expected distributions $(P>0.05)$. In the case of chr4:153210505-153212191, the frequency of deletion (or loss) in the case group was higher than that in the control group. This fact suggests that the deletion allele may be a risk factor for SAH. In the case of chr10:6265006-6267388, the frequency of duplication (or gain) in the case group was higher than that in the control group. This fact indicates that the increase in copy number in the region may influence the onset of SAH. These two regions contained one gene and four nearby genes within $500 \mathrm{~kb}$. Moreover, we observed that these two regions overlapped with the previous identified CNVs on DGV (Supplementary Figure 3). 
Table 1 Summary of common CNV regions (frequency $>5 \%$ )

\begin{tabular}{|c|c|c|c|c|c|c|}
\hline$C N V$ region & Length (bp) & $\begin{array}{l}\text { No. of } \\
\text { CNVs }\end{array}$ & Frequency & $\begin{array}{l}\text { No. of } \\
\text { genes }\end{array}$ & Gene & $\begin{array}{l}\text { Overlapped } \\
\text { with DGVa }\end{array}$ \\
\hline chr18:64877649-64909977 & 32328 & 149 & 0.314 & 0 & & Yes \\
\hline chr4:161993023-162154792 & 161769 & 85 & 0.179 & 0 & & Yes \\
\hline chr3:65166887-65187636 & 20749 & 59 & 0.124 & 0 & & Yes \\
\hline chr11:49483244-51297186 & 1813942 & 52 & 0.109 & 6 & $\begin{array}{l}\text { LOC44004O,LOC441601,LOC646813, } \\
\text { OR4A5,OR4C12,OR4C13 }\end{array}$ & Yes \\
\hline chr14:43524894-43584372 & 59478 & 38 & 0.080 & 0 & & Yes \\
\hline chr19:642025-1523369 & 881344 & 37 & 0.078 & 42 & $\begin{array}{l}\text { ABCA7,ADAMTSL5,APC2,ARID3A,ATP5D,AZU1,C19orf21, } \\
\text { C19orf22,C19orf23,C19orf24,C19orf25,C19orf26,C19orf6, } \\
\text { CFD,CIRBP,CNN2,DAZAP1,EFNA2,ELA2,GAMT,GPX4,GRIN3B, } \\
\text { HMHA1,KISS1R,MED16,MEX3D,MIDN,MUM1,NDUFS7,PALM, } \\
\text { PCSK4,PLK5P,POLR2E,PRG2,PRSSL1,PRTN3,PTBP1,REEP6, } \\
\text { RPS15,SBNO2,STK11,WDR18 }\end{array}$ & Yes \\
\hline chr12:130374756-130398112 & 23356 & 36 & 0.076 & 0 & & Yes \\
\hline chr5:41608479-41632026 & 23547 & 35 & 0.074 & 0 & & Yes \\
\hline chr7:153118877-153284978 & 166101 & 34 & 0.072 & 1 & DPP6 & Yes \\
\hline chr15:45994758-46034246 & 39488 & 32 & 0.067 & 0 & & Yes \\
\hline chr21:16683912-16708090 & 24178 & 31 & 0.065 & 1 & C21orf34 & Yes \\
\hline chr8:5316404-5947152 & 630748 & 30 & 0.063 & 0 & & Yes \\
\hline chr6:29188328-29432578 & 244250 & 29 & 0.061 & 5 & LOC651503,OR14J1,OR2J2,OR2J3,OR5V1 & Yes \\
\hline chr7:3411837-3431422 & 19585 & 29 & 0.061 & 1 & $S D K 1$ & Yes \\
\hline chr21:26116740-26163866 & 47126 & 26 & 0.055 & 0 & & Yes \\
\hline chr8:145050595-145299088 & 248493 & 26 & 0.055 & & $\begin{array}{l}\text { C8orf3OA,CYC1, EXOSC4, GPAA1, GRINA, HEATR7A, } \\
\text { KIAA1875, MAF1,OPLAH,PARP1O,PLEC1,SHARPIN,SPATC1 }\end{array}$ & Yes \\
\hline
\end{tabular}

Abbreviations: CNV, copy number variation; DGV, Database of Genomic Variant.

aDatabase of Genomic Variants (http://projects.tcag.ca/variation).

Table 2 Logistic regression analysis of identified CNV with risk of subarachnoid aneurysmal hemorrhage while controlling age and gender as covariates among case and normal subjects

\begin{tabular}{|c|c|c|c|c|c|c|c|c|c|c|c|c|c|c|c|c|c|}
\hline \multirow[b]{2}{*}{$\begin{array}{l}\text { CNV } \\
\text { type }\end{array}$} & \multirow[b]{2}{*}{ CNV region ${ }^{\mathrm{a}}$} & \multirow[b]{2}{*}{ Cytoband } & \multirow[b]{2}{*}{$\begin{array}{c}\text { Length } \\
\text { (kb) }\end{array}$} & \multirow[b]{2}{*}{$M A F$} & \multicolumn{3}{|c|}{ P-value (HWE) } & \multicolumn{3}{|c|}{ Case } & \multicolumn{3}{|c|}{ Control } & \multirow[b]{2}{*}{ P-value } & \multirow[b]{2}{*}{$\mathrm{P}^{\text {corr b }}$} & \multirow[b]{2}{*}{ Gene } & \multirow[b]{2}{*}{$\begin{array}{l}\text { Nearby } \\
\text { genes (kb) }\end{array}$} \\
\hline & & & & & $A / l$ & $\begin{array}{l}\text { Cases } \\
(\mathrm{n}=191)\end{array}$ & $\begin{array}{l}\text { Controls } \\
(\mathrm{n}=282)\end{array}$ & $\begin{array}{l}L / L \\
(O X)\end{array}$ & $\begin{array}{l}L / N \\
(1 X)\end{array}$ & $\begin{array}{l}N / N \\
(2 X)\end{array}$ & $\begin{array}{l}L / L \\
(O X)\end{array}$ & $\begin{array}{l}L / N \\
(1 X)\end{array}$ & $\begin{array}{l}N / N \\
(2 X)\end{array}$ & & & & \\
\hline \multirow[t]{4}{*}{ Loss } & chr4: & $4 q 31.3$ & 1.7 & 0.019 & 0.673 & 0.572 & 0.928 & 0 & 15 & 176 & 0 & 3 & 279 & 0.0005 & 0.04 & & PET112 L \\
\hline & 153210505- & & & & & & & $G / G$ & $G / N$ & $N / N$ & $G / G$ & $G / N$ & $N / N$ & & & & (308.9), \\
\hline & 153212191 & & & & & & & $(>4 X)$ & $(3 X)$ & $(2 X)$ & $(>4 X)$ & $(3 X)$ & $(2 X)$ & & & & FBXW7 \\
\hline & & & & & & & & & & & & & & & & & (249.7) \\
\hline \multirow[t]{3}{*}{ Gain } & $\begin{array}{l}\text { chr10: } \\
\text { 6265006- }\end{array}$ & 10p15.1 & 2.4 & 0.01 & 0.835 & 0.768 & 0.976 & 0 & 8 & 183 & 0 & 1 & 281 & 0.0006 & 0.05 & PFKFB3' & $\begin{array}{l}\text { RBM 17 } \\
(65.6)\end{array}$ \\
\hline & 6267388 & & & & & & & & & & & & & & & & PFKFB3 \\
\hline & & & & & & & & & & & & & & & & & $(17.5)^{d}$ \\
\hline
\end{tabular}

Abbreviations: CNV, copy number variation; G/G, gain/gain; G/N, gain/normal; HWE, Hardy-Weinberg equilibrium; LD, linkage disequilibrium; L/L, loss/loss; L/N, loss/normal; MAF, minor allele frequency; N/N, normal/normal; N/N, normal/normal; $P^{\text {corr, }}$ corrected $P$-value.

Bold values indicate the case of $P<0.05$.

aVersion: Human Mar. 2006 (NCBI36/hg18) Assembly.

bTo achieve the optimal correction for multiple testing of markers representing common CNVs (frequency $>1 \%$ ) in LD with each other, the effective number of independent marker loci (89.3639) was calculated using the software SNPSpD (http://genepi.qimr.edu.au/general/daleN/SNPSpD/), on the basis of spectral decomposition (SpD) of matrices of pairwise LD between the markers.

${ }^{c}$ Homo sapiens 6-phosphofructo-2-kinase/fructose-2,6-biphosphatase 3 (PFKFB3), transcript variant 2, NCBI Reference Sequence: NM_001145443.

${ }^{\mathrm{d}}$ Homo sapiens 6-phosphofructo-2-kinase/fructose-2,6-biphosphatase 3 (PFKFB3), transcript variant 1, NCBI Reference Sequence: NM_004566. 
a

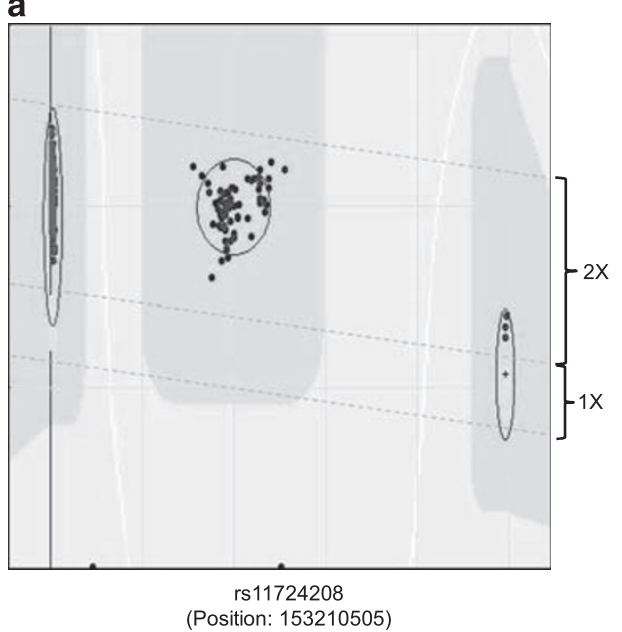

b

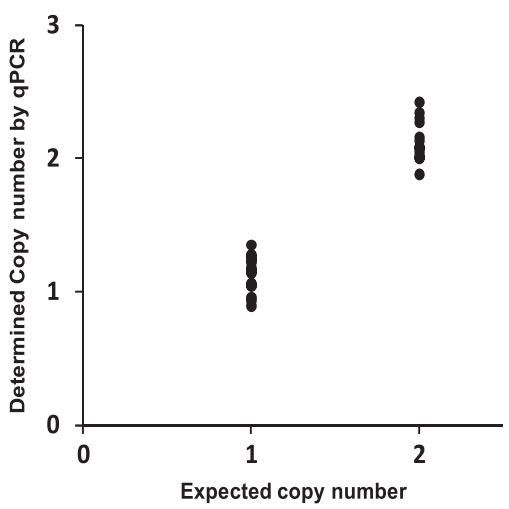

Figure 1 Copy number variation validation by quantitative PCR (qPCR) around rs11724208 within chr4:153210505-153212191. (a) Genoplot image of identified deletions (marker name: rs11724208). One genoplot image represents allelic intensity ( $x$ axis) and signal intensity ( $y$ axis) of all samples. Two types of copy number ( $2 \mathrm{X}$ and $1 \mathrm{X}$ ) are depicted. Individuals having hemizygous deletions (copy number: $1 \mathrm{X}$ ) clustered into two distinct groups (color: yellow). Samples having null copy number are displayed with a black dot at the bottom. (b) Validation by qPCR around the rs11724208 within chr4:153210505153212191. The value of $x$ axis (expected copy number) was estimated using the Illumina Genoplot image analysis. The $y$ axis indicates the determined copy number by qPCR. The copy number value estimated through visual examination matched with the quantitative measurement value using qPCR. A full color version of this figure is available at the Journal of Human Genetics journal online.

Figure 1 shows a visualization of identified CNV (CNV type: deletion) within chr4:153210505-153212191 in UCSC Genome Browser and Illumina genoplot images. This genoplot image, which represents signal and allelic intensity of samples per marker, shows that the hemizygous deletions in the first marker (rs11724208; position: 153210505) were also observed in the third marker (rs10029800; position: 153212191).

\section{DISCUSSION}

SAH due to rupture of cerebral aneurysm accounts for $6-8 \%$ of total stroke cases. As $2-5 \%$ of the world's total population has cerebral aneurysm, it is necessary to broaden the study on factors that cause SAH. Early precaution and treatment are very important, as $40 \%$ of patients with SAH may die during the onset of the disease.

In this study, we hypothesized that $\mathrm{CNV}$ is significantly associated with the risk of SAH. To perform association analysis, it is important to accurately identify individual CNVs. For this purpose, we used pennCNV, a program that is optimized for SNP genotyping BeadChip using multiple factors, namely, signal intensity, allelic intensity and population frequency of $\mathrm{B}$ allele. ${ }^{17,22}$ This program has been widely used by researchers and the results generated from the program are already publicized in papers. ${ }^{22-27}$ Two other ways of verifying the accuracy of the identified CNVs involve comparing the results with the DGV databases in which information on previously reported CNVs is stored, or using another CNV identification program to compare the results with.

To identify reliable CNVs, we used the Illumina HumanHap300 BeadChip, which contains 317503 SNP/CNV markers. We also compared the results of the individual CNVs that were identified in this study with that of the DGV database and with another CNV identification program called CNVpartition. Our findings revealed that the CNVs identified in this study were in concordance with the DGV database at $73.2 \%$ and with the CNVpartition at $72.4 \%$ (data not shown), an indication of high-quality CNVs. After comparing the individual CNVs and segmental duplications, we found that many $\mathrm{CNVs}$ and common $\mathrm{CNV}$ regions (>5\%) were located in the segmental duplication region (33.9 and 45.5\%, respectively). This observation is similar to the results of other studies $6,28,29$ wherein $\mathrm{CNV}$ s discovered were closely related with segmental duplication. ${ }^{14}$ Further, results from GO analysis showed that cell differentiation displayed distributions similar to those of previous studies. However, sensory perception of smell and neurophysiological processes did not produce a highly enriched region in the GO category (Supplementary Table 2).

To identify disease-susceptible regions for the risk of SAH, we performed a genome-wide CNV association analysis using a logistic regression model. In this study, we found two new CNV regions (chr4:153210505-153212191 and chr10:6265006-6267388) that were significantly associated with risk of SAH. Interestingly, we also observed that PET112L (PET112, Saccharomyces cerevisia, homologlike), FBXW7 (F-box and WD40 domain protein 7), PFKFB3 (transcript variant 1 of 6-phosphofructo-2-kinase/fructose-2,6-biphosphatase 3) and RBM17 (RNA binding motif protein 17) genes were located within $500 \mathrm{~kb}$ of those CNV regions. PET112L (OMIM no.: 603645) is predominantly expressed in muscle and heart tissues in which oxidative phosphorylation occurs at a high rate. Although this protein has a role in accumulation of cytochrome oxidase subunit II and in the stability of mitochondrial genome in S. cerevisia, ${ }^{30}$ its function in the human body is still unclear. FBXW7 (OMIM no.: 606278), on the other hand, targets the ligase activity to cyclin E, a protein previously implicated in the regulation of neuronal apoptosis. Reciprocal relation with loss of FBXW7 and deletion or mutation of PTEN has been reported to be associated with human breast cancer cell lines and primary tumors. ${ }^{31}$ Furthermore, both RBM17 and PFKFB3 genes have been considered to be associated with diabetes, obesity and endocrine disease; ${ }^{32}$ however, further studies are required to clarify how those genes may affect an individual's susceptibility to SAH. From our previous study, we found that one SNP marker (rs1242541) within a CNV region was significantly associated with the risk of SAH $(P=0.0006),{ }^{13}$ but in this study, no significant association was detected in this region. These discrepancies in the results may be due to the fact that our previous study only analyzed single multiallelic 
CNV markers on CNV regions. In other words, the present study, therefore, is different from the previous study, as analyses of CNVs were conducted at a larger scale.

In conclusion, we have described the first genome-wide CNV association analysis on $\mathrm{CNV}$ and the risk of SAH. We found significant associations between several CNV regions and the risk of SAH after identifying 4574 individual CNVs from 473 samples using the Illumina HumanHap300 BeadChip. Although future studies should be conducted to determine those susceptible regions that are functional and elaborate on the underlying mechanism of their function, our findings suggest that the newly identified disease susceptible CNV regions in this study may influence the risk of SAH in a Japanese population.

\section{CONFLICT OF INTEREST}

The authors declare no conflict of interest.

\section{ABBREVIATIONS}

CNV, copy number variation; DGV, Database of Genomic Variant; SAH, subarachnoid aneurysmal hemorrhage.

\section{ACKNOWLEDGEMENTS}

We thank Dr Kai Wang (Department of Genetics, University of Pennsylvania, and Center for Applied Genomics, Children's Hospital of Philadelphia, Philadelphia, PA 19104, USA) for providing useful scripts for the pennCNV. This work was supported by the National Research Foundation of Korea (NRF) grant funded by the Korea government (MEST) (no. 2009-0080157). This research was supported by the Basic Science Research Program through the National Research Foundation of Korea (NRF) funded by the Ministry of Education, Science and Technology (no. 2010-0011206). This work was also supported by Priority Research Centers Program through the National Research Foundation of Korea (NRF) funded by the Ministry of Education, Science and Technology (no. 2009-0093822).

1 Hop, J. W., Rinkel, G. J., Algra, A. \& van Gijn, J. Case-fatality rates and functional outcome after subarachnoid hemorrhage: a systematic review. Stroke 28, 660-664 (1997).

2 Yong-Zhong, G. \& van Alphen, H. A. Pathogenesis and histopathology of saccular aneurysms: review of the literature. Neurol. Res. 12, 249-255 (1990).

3 Bromberg, J. E., Rinkel, G. J., Algra, A., Greebe, P., van Duyn, C. M., Hasan, D. et al. Subarachnoid haemorrhage in first and second degree relatives of patients with subarachnoid haemorrhage. Br Med J 311, 288-289 (1995).

4 Ruigrok, Y. M., Buskens, E. \& Rinkel, G. J. Attributable risk of common and rare determinants of subarachnoid hemorrhage. Stroke 32, 1173-1175 (2001).

5 Cook, E. H. Jr. \& Scherer, S. W. Copy-number variations associated with neuropsychiatric conditions. Nature 455, 919-923 (2008)

6 Redon, R., Ishikawa, S., Fitch, K. R., Feuk, L., Perry, G. H., Andrews, T. D. et al. Global variation in copy number in the human genome. Nature 444, 444-454 (2006).

7 Freeman, J. L., Perry, G. H., Feuk, L., Redon, R., McCarroll, S. A., Altshuler, D. M. et al. Copy number variation: new insights in genome diversity. Genome Res. 16, 949-961 (2006).

8 Henrichsen, C. N., Vinckenbosch, N., Zollner, S., Chaignat, E., Pradervand, S., Schutz, F. et al. Segmental copy number variation shapes tissue transcriptomes. Nat. Genet. 41, 424-429 (2009)

9 Sebat, J., Lakshmi, B., Malhotra, D., Troge, J., Lese-Martin, C., Walsh, T. et al. Strong association of de novo copy number mutations with autism. Science 316, 445-449 (2007).
10 Cappuzzo, F., Hirsch, F. R., Rossi, E., Bartolini, S., Ceresoli, G. L., Bemis, L. et al. Epidermal growth factor receptor gene and protein and gefitinib sensitivity in nonsmall-cell lung cancer. J. Natl Cancer Inst. 97, 643-655 (2005).

11 St Clair, D. Copy number variation and schizophrenia. Schizophr. Bull. 35, 9-12 (2009).

12 Knight, S. J., Regan, R., Nicod, A., Horsley, S. W., Kearney, L., Homfray, T. et al. Subtle chromosomal rearrangements in children with unexplained mental retardation. Lancet 354, 1676-1681 (1999).

13 Bae, J. S., Cheong, H. S., Kim, J. O., Lee, S. O., Kim, E. M., Lee, H. W. et al. Identification of SNP markers for common CNV regions and association analysis of risk of subarachnoid aneurysmal hemorrhage in Japanese population. Biochem. Biophys. Res. Commun. 373, 593-596 (2008).

14 Kathiresan, S., Voight, B. F., Purcell, S., Musunuru, K., Ardissino, D., Mannucci, P. M. et al. Genome-wide association of early-onset myocardial infarction with single nucleotide polymorphisms and copy number variants. Nat. Genet. 41, 334-341 (2009).

15 Need, A. C., Attix, D. K., McEvoy, J. M., Cirulli, E. T., Linney, K. L., Hunt, P. et al. A genome-wide study of common SNPs and CNVs in cognitive performance in the CANTAB. Hum. Mol. Genet. 18, 4650-4661 (2009).

16 Makishima, T., Nakashima, T., Nagata-Kuno, K., Fukushima, K., lida, H., Sakaguchi, M. et al. The highly conserved DAD1 protein involved in apoptosis is required for N-linked glycosylation. Genes Cells 2, 129-141 (1997).

17 Wang, K., Li, M., Hadley, D., Liu, R., Glessner, J., Grant, S. F. et al. PennCNV: an integrated hidden Markov model designed for high-resolution copy number variation detection in whole-genome SNP genotyping data. Genome Res. 17, 1665-1674 (2007).

18 Wang, K., Chen, Z., Tadesse, M. G., Glessner, J., Grant, S. F., Hakonarson, H. et al. Modeling genetic inheritance of copy number variations. Nucleic. Acids Res. 36, e138 (2008).

19 Nyholt, D. R. A simple correction for multiple testing for single-nucleotide polymorphisms in linkage disequilibrium with each other. Am. J. Hum. Genet. 74, 765-769 (2004).

20 Beissbarth, T. Interpreting experimental results using gene ontologies. Methods Enzymol. 411, 340-352 (2006).

21 Beissbarth, T. \& Speed, T. P. GOstat: find statistically overrepresented Gene Ontologies within a group of genes. Bioinformatics 20, 1464-1465 (2004).

22 Wang, K., Zhang, H., Ma, D., Bucan, M., Glessner, J. T., Abrahams, B. S. et al. Common genetic variants on 5p14.1 associate with autism spectrum disorders. Nature 459, 528-533 (2009).

23 Cusco, I., Medrano, A., Gener, B., Vilardell, M., Gallastegui, F., Villa, O. et al. Autismspecific copy number variants further implicate the phosphatidylinositol signaling pathway and the glutamatergic synapse in the etiology of the disorder. Hum. Mol. Genet. 18, 1795-1804 (2009).

24 Daly, A. K., Donaldson, P. T., Bhatnagar, P., Shen, Y., Pe'er, I., Floratos, A. et al. HLA$B * 5701$ genotype is a major determinant of drug-induced liver injury due to flucloxacillin. Nat. Genet. 41, 816-819 (2009).

25 Diskin, S. J., Hou, C., Glessner, J. T., Attiyeh, E. F., Laudenslager, M., Bosse, K. et al. Copy number variation at 1q21.1 associated with neuroblastoma. Nature 459, 987-991 (2009).

26 Jakobsson, M., Scholz, S. W., Scheet, P., Gibbs, J. R., VanLiere, J. M., Fung, H. C. et al. Genotype, haplotype and copy-number variation in worldwide human populations. Nature 451, 998-1003 (2008).

27 Vrijenhoek, T., Buizer-Voskamp, J. E., van der Stelt, I., Strengman, E., Sabatti, C., Geurts van Kessel, A. et al. Recurrent CNVs disrupt three candidate genes in schizophrenia patients. Am. J. Hum. Genet. 83, 504-510 (2008).

28 Cooper, G. M., Nickerson, D. A. \& Eichler, E. E. Mutational and selective effects on copy-number variants in the human genome. Nat. Genet. 39, S22-S29 (2007).

29 Perry, G. H., Tchinda, J., McGrath, S. D., Zhang, J., Picker, S. R., Caceres, A. M. et al. Hotspots for copy number variation in chimpanzees and humans. Proc. Natl Acad. Sci. USA 103, 8006-8011 (2006).

30 Petruzzella, V., Tiranti, V., Fernandez, P., lanna, P., Carrozzo, R. \& Zeviani, M. Identification and characterization of human cDNAs specific to BCS1, PET112, SCO1, COX15, and COX11, five genes involved in the formation and function of the mitochondrial respiratory chain. Genomics 54, 494-504 (1998).

31 Mao, J. H., Kim, I. J., Wu, D., Climent, J., Kang, H. C., DelRosario, R. et al. FBXW7 targets mTOR for degradation and cooperates with PTEN in tumor suppression. Science 321, 1499-1502 (2008)

32 Lowe, C. E., Cooper, J. D., Brusko, T., Walker, N. M., Smyth, D. J., Bailey, R. et al. Large-scale genetic fine mapping and genotype-phenotype associations implicate polymorphism in the IL2RA region in type 1 diabetes. Nat. Genet. 39, 1074-1082 (2007).

Supplementary Information accompanies the paper on Journal of Human Genetics website (http://www.nature.com/jhg) 